\title{
Bridging molecular and continuous descriptions: the case of dynamics in clays
}

\author{
JEAN-FRANÇOIS DUFRÊCHE ${ }^{1,2}$, BENJAMIN ROTENBERG ${ }^{1,3}$, VIRGINIE MARRY ${ }^{1}$ and PIERRE TURQ TI,2 $^{1,2}$ \\ ${ }^{1}$ UMR PECSA, UPMC-Paris 6, CNRS, 4 place Jussieu, 75252 Paris Cedex 05, France \\ ${ }^{2}$ ICSM UMR 5257 CEA/CNRS/Université de Montpellier 2/ENSCM, Centre de Marcoule Bât. 426 BP 17171 \\ 30207 Bagnols sur Cèze Cedex, France \\ ${ }^{3}$ ANDRA, Parc de la Croix Blanche, 1/7 rue Jean Monnet, 92298 Châtenay Malabry Cedex, France \\ Manuscript received on April 25, 2008; accepted for publication on July 27, 2008
}

\begin{abstract}
The theory of transport in porous media such as clays depends on the level of description. On the macroscopic scale, hydrodynamics equations are used. These continuous descriptions are convenient to model the fluid motion in a confined system. Nevertheless, they are valid only if the pores of the material are much larger than the molecular size of the components of the system. Another approach consists in using molecular descriptions. These two methods which correspond to different levels of description are complementary. The link between them can be clarified by using a coarse-graining procedure where the microscopic laws are averaged over fast variables to get the long time macroscopic laws. We present such an approach in the case of clays. Firstly, we detail the various levels of description and the relations among them, by emphasizing the validity domain of the hydrodynamic equations. Secondly, we focus on the case of dehydrated clays where hydrodynamics is not relevant. We show that it is possible to derive a simple model for the motion of the cesium ion based on the difference on time scale between the solvent and the solute particles.
\end{abstract}

Key words: clays, multi-scale modelling, molecular dynamics, hydrodynamics, Brownian model.

\section{INTRODUCTION}

Clays are multi-scale materials. The description of the dynamics of such complex systems is to be made at different scales. On the macroscopic scale, hydrodynamics equations are very convenient to model the fluid motion and the diffusion of the various species through this porous medium. Most of the time, the NavierStokes equation is coupled to the Poisson equation and the Fick's law in order to compute the macroscopic properties of the system as a function of the geometry. Yet the link among these macroscopic laws and the microscopic properties of the material has to be clarified.

Selected paper presented at the IUTAM Symposium on Swelling and Shrinking of Porous Materials: From Colloid Science to Poromechanics - August 06-10 2007, LNCC/MCT.

Correspondence to: Jean-François Dufrêche

E-mail: jean-francois.dufreche@upmc.fr
They are valid only if the pores of the material are very large, but the exact condition has to be determined. In addition, macroscopic parameters (zeta potential, effective viscosity, diffusion coefficients, adsorption coefficients) are required.

Further approaches are based on molecular descriptions. With the development of computer science, it is possible to perform molecular dynamics simulations in the case of such a confined system. The precise motion of the particles is obtained at short time and short length scales. In that case the difficulty arises from the fact that the boundary conditions of the simulation do not correspond to the exact experimental system since long-scale porosities are ignored and the link with the experiments has to be clarified.

Thus the two methods are complementary since they allow to describe the complex structure of the 
material. At short scales molecular dynamics can be used whereas macroscopic laws are required at large scales. The link between the descriptions still requires further developments. Rigorous strategy can be obtained if one uses coarse-graining procedures where the microscopic laws are averaged over fast variables to get the long time macroscopic laws. We present such approaches in the case of clays.

First, we will focus on hydrated clays. We will briefly introduce the various levels of description that can be used in order to describe montmorillonite clays. Comparison between molecular dynamics and macroscopic laws allows the determination of the validity domain of commonly used theories, such as the PoissonBoltzmann equation. In the case of hydrodynamics, the comparisons show that the macroscopic laws are valid if slip boundary conditions are taken into account.

Second, the case of ionic diffusion for dehydrated clays is analyzed. In that case, the classical equations (e.g. Nernst-Planck model) are not valid any more because of the size of the pore. Continuous solvent models based on bulk equation are not valid because of the layering of the solvent, which depends on its molecular structure. We have introduced a coarse-graining procedure, which generalizes the latter to the case of confined systems. We take into account the molecular structure of the water to compute (i) the interactions between the particles and (ii) their mobility. These preliminary calculations show that it is possible to describe the motion of the ions in a continuous solvent model, even in the case of dehydrated clays, provided that the mass of the ion is much larger than that of the solvent (cesium ion e.g.).

\section{MULTI-SCALE MODELLING}

\section{LEVELS OF DESCRIPTIONS}

Hydrated smectite clays such as montmorillonite are lamellar mineral crystals composed of charged layers separated by an aqueous solution. They exhibit special features towards hydration and ion fixation (Karaborni et al. 1996). Clay lamellae form thin platelet-shaped particles of diameter close to several hundreds of Ångströms. However this lamellar geometry is valid only at small length scales. At larger scales, the structure is more complex and it leads to multi-porosities. Thus the de- scription of such systems requires a multi-scale strategy. Many theoretical studies at different levels of description have been performed. They are summarized in Figure 1.

Ab-initio molecular dynamics (Boek and Sprik 2003, Tunega et al. 2004, Benco et al. 2001) provides information about the electronic degrees of freedom, but because of its computational cost it is restricted to the smallest time and length scales. Classical MonteCarlo (Skipper et al. 1995) or Molecular Dynamics simulations (Malikova et al. 2004, Arab et al. 2004, Greathouse et al. 2000) are able to describe larger systems. For example, the mechanism of crystalline swelling at low hydration is well reproduced by these techniques where the various atoms and ions are considered explicitly. Nevertheless, very large systems (e.g. for high hydration, or when different platelets have to be taken into account) cannot be treated by this technique. The use of alternative methods such as Brownian dynamics simulations (Marry et al. 2003) is inescapable. In that case, only a part of the degrees of freedom (e.g. the motion of the (free) ions) is considered. At large scales, continuous methods (e.g. Poisson-Boltzmann (Trizac et al. 2000) descriptions or hydrodynamics (Rosanne et al. 2004, Leroy and Revil 2004, Moyne and Murad 2002, Moyne and Murad 2003)) can model the different sort of porosities. They are especially relevant for the derivation of the macroscopic laws (such as Darcy's law) or for the calculation of the various electrokinetic phenomena.

\section{ELIMINATION OF FAST VARIABLES}

Unfortunately, the link among these methods is at first sight far from obvious. Yet this issue is very important since every method requires the knowledge of the parameters of the model it is based on. These parameters could be obtained - at least in principle - from the simulations at the lower level of description. This self-consistent approach is not commonly used for clays, though. Most of the numerical studies obtain their parameter from experiments or from semi-phenomenological considerations. Only a few attempts have been made towards that direction for clays (Marry et al. 2003, Porion et al. 2003).

The general method, which bridges the gap among the various models, is the elimination of fast variables (Van Kampen 1985). Every level of description can be obtained from the preceding one by averaging over 


\begin{tabular}{|c|c|c|c|c|}
\hline $\begin{array}{l}\text { Ab Initio (Quantum) } \\
\text { Molecular Dynamics }\end{array}$ & $\begin{array}{c}\text { Classical } \\
\text { Molecular Dynamics }\end{array}$ & Brownian Dynamics & $\begin{array}{c}\text { Hydrodynamics } \\
\text { Non Eq. Thermodynamics }\end{array}$ & $\begin{array}{c}\text { Porous Media } \\
\text { Hydrodynamics }\end{array}$ \\
\hline $\begin{array}{c}\text { Nuclei } \\
\text { Electrons (density) }\end{array}$ & $\begin{array}{l}\text { Atoms Molecules } \\
\text { Ions }\end{array}$ & $\begin{array}{l}\text { Ions (Solute Particles) } \\
\text { Continuous Solvent }\end{array}$ & $\begin{array}{l}\text { Continuous Description } \\
\text { Navier-Stokes, Fick } \\
\text { Poisson-Bolzmann Eqs. }\end{array}$ & $\begin{array}{r}\text { Pore Averaged Laws } \\
\text { (e.g.: Darcy's Law) }\end{array}$ \\
\hline
\end{tabular}

Fig. 1 - Various levels of description for the dynamics in charged porous systems. Upper part: simulation and theoretical techniques. Lower part: ingredients of the models.

some degrees of freedom. Then it only describes the dynamics of a part of the degrees of freedom (relevant variables). Further degrees of freedom (irrelevant variables) are not described explicitly at the considered level. They are only included via their average effect. For example, in the case of molecular dynamics, the only relevant variables are the position of the nuclei. The details of the electronic degrees of freedom are ignored. Only their average effects, i.e. the forces on the nuclei are taken into account. This separation among the variables makes sense for the calculation of the dynamical properties only if the irrelevant variables are much faster than the relevant ones. Thus, such a coarse-grained procedure is valid if slow variables coincide with relevant ones. In the case of molecular dynamics, it corresponds to the Born-Oppenheimer approximation that simplifies the calculation of the motion of the nuclei under the influence of the rapid motion of the electrons.

Similarly, in the case of Brownian dynamics, the motion of the solute particles (i.e. ions) is obtained by considering that the only relevant variables are the degrees of freedom of the solute particles. This Brownian description which treats the solvent as a continuum is valid only if the solvent molecules are much faster than the solute particles.

The elimination of the fast variables is the key for the derivation of the various models of Figure 1, except for the last one. Indeed, the experimental macroscopic law of porous media (such as Darcy's law) are theoretically obtained differently, by averaging over the geometry of the material, for example thanks to the homogenization procedure of Moyne and Murad (Moyne and Murad 2002, 2003).

\section{HYDRODYNAMIC LIMIT}

It should be noted that the hydrodynamic equations themselves correspond to the elimination of fast vari- ables. Thus the laws of non-equilibrium thermodynamics (de Groot and Mazur 1983) such as the NavierStokes equation for the convection and the Fick's law for the diffusive motion of the solute particles, are valid in the hydrodynamic limit (Ailawadi et al. 1971, Zwanzig 2001) where the hydrodynamic modes become slow. The latter corresponds to the limit of large distances $r$ or small wave-vectors $k$, as can be understood from the following argument.

Non-equilibrium thermodynamics predicts the evolution of particle and momentum distributions in space. For example, in the case of diffusion, it gives the evolution of the density of particles in space for the various components of the systems. The microscopic density of a species $\alpha$ reads:

$$
\rho_{\alpha}(\mathbf{r}, t)=\sum_{i} \delta\left(\mathbf{r}-\mathbf{r}_{i}(t)\right)
$$

where the $i$ th particle of type $\alpha$ has position $\mathbf{r}_{i}(t)$ at time $t$. The Fourier transform is:

$$
\rho_{\alpha}^{\star}(\mathbf{k}, t)=\int \rho_{\alpha}(t) e^{-i \mathbf{k} \cdot \mathbf{r}} \mathrm{d} \mathbf{r}=\sum_{i} e^{-i \mathbf{k} \cdot \mathbf{r}_{i}(t)}
$$

In the hydrodynamic limit $\mathbf{k} \rightarrow 0, \rho_{\alpha}^{\star}(\mathbf{k}, t)$ becomes time-independent because it tends towards $N_{\alpha}$, the total number of particles of type $\alpha$. For $\mathbf{k} \neq 0, \rho_{\alpha}^{\star}(\mathbf{k}, t) \neq N_{\alpha}$ depends on time. But its evolution gets slower as $\mathbf{k} \rightarrow$ 0 . Thus for $\mathbf{k} \rightarrow 0$, the density of particle becomes a slow variable. A similar argument can be made for the average velocity (or momentum) of the particles:

$$
\mathbf{v}(\mathbf{r}, t)=\frac{1}{\sum_{i} m_{i}} \sum_{i} \delta\left(\mathbf{r}-\mathbf{r}_{i}(t)\right) m_{i} \mathbf{v}_{i}(t)
$$

because of the linear momentum conservation law. Thus in the hydrodynamic limit (small wave-vectors or large distances), hydrodynamic laws are valid because the hydrodynamic quantities become slow, on the grounds of the conservation laws. 
The accuracy of hydrodynamic descriptions can be checked by comparing their predictions to the results obtained from molecular dynamics (Marry and Turq 2003, Dufrêche et al. 2005b). Some of the results are given in Figure 2 for Na montmorillonite clays with an interlayer spacing equal to $47.5 \AA$. In that case, macroscopic hydrodynamic models generally calculate counterion distributions with the help of the PoissonBoltzmann equation. Even for that relatively small interlayer spacing, this hydrodynamic model appears to be valid. The main differences are the oscillations given by the microscopic model, which are characteristic of the discrete nature of the solvent molecules. The electroosmotic flow can be calculated from molecular dynamics as well. In that case simple hydrodynamic theory fails to reproduce the microscopic results. In fact, it seems that the problem comes from the stick boundary conditions. One can show that microscopically, slip boundary conditions have to be taken into account. More precisely, the parallel component of the hydrodynamic fluid velocity at the interface is not zero. It is actually given by:

$$
v_{p}=\delta \frac{\partial v_{p}}{\partial z}
$$

where $z$ is the direction orthogonal to the interface and $\delta$ is the Navier slip length that quantifies slipping. For montmorillonite, $\delta$ is equal to a few Ångströms, which is very small, but it is enough to modify the electroosmotic flow. The reason comes from the fact that electro-osmosis is created by surface charges, so that it strongly depends on the boundary conditions. Conversely, hydrodynamic motions created by bulk forces (such as a pressure gradient in the case of a Poiseuille flow or of a streaming potential experiment) depend less on the slip length, as it has been recently confirmed by Joly et al. (Joly et al. 2006).

\section{DEHYDRATED CLAYS}

At very low interlayer spacings, hydrodynamic models inside the pores do not hold anymore. For example, in the important case of monohydrated clays, there is only one layer of water molecules between two clay sheets, so there is no chance that hydrodynamic equations work. Yet it is not impossible to derive a continuous solvent model for the dynamics of the ions in that case. Indeed, the hydrodynamic quantities are not slow anymore, because the distance between the layers is not large enough, but if the ions are much heavier than the water molecules, their dynamics is much slower than the dynamics of the solvent. Thus, the position and the velocity of the solute particles are slow variables and a coarse-graining procedure can be performed. The motion of the ions is given by a Brownian model. The diffusion of heavy ions in clays at low hydration is particularly important because of the practical applications. In particular, it corresponds to the case of nuclear waste storage.

\section{BROWNIAN MODEL}

Most theories of transport of electrolyte solutions, which generalized the pioneering work of Onsager (Onsager and Fuoss 1932) are actually based on Brownian description. At first sight it may be surprising that Brownian theories can be applied for simple ions that are not quite heavier than the solvent molecules. However, in practice, for bulk solutions, the model is satisfactory even for small ions as $\mathrm{Li}^{+}$, probably because of the phenomenon of hydration. A shell of solvent molecules becomes strongly attached to each ion so that it is probably not too bad an approximation to consider a given ion together with its solvation shell as a single heavy entity.

Different Brownian models can be implemented for the dynamics of ions in an aqueous solution. They are summarized in Figure 3. At the microscopic scale, discrete solvent descriptions consider all the different particles, the solvent and the ions. The dynamics (Hansen and McDonald 1986) is driven by Newton's law (which corresponds to the Liouville's equation in phase space). If solute particles can be considered to be much heavier than the solvent molecules, Brownian models (based on continuum solvent descriptions) are valid. There are mainly two classes of Brownian description (Résibois 1968). The first one corresponds to the Langevin approach. The relevant observables are the velocity (or the momentum) and the position of the ions. The evolution in phase space is given by a Fokker-Planck equation, as it can be shown by a Kramers Moyal expansion (Risken 1996, Van Kampen 1992). The second one is the Smoluchowski approach. It is valid for time scales longer than the relaxation time $\tau_{\mathrm{v}}=1 / \gamma$ of the velocities of 




Fig. 2 - Left: Cation $\left(\mathrm{Na}^{+}\right)$distribution between two montmorillonite clay layers. Solid line: molecular dynamics. Dashed line: Poisson-Boltzmann equation. Right: Electro-osmotic flux. Diamonds: molecular dynamics. Dashed line: Navier-Stokes equation with stick boundary condition. Solid line: Navier-Stokes equation with slip boundary conditions ( $\delta=6 \AA$ ).

\begin{tabular}{|c|c|c|c|c|}
\hline Model & $\begin{array}{l}\text { Microscopic } \\
\text { Variables }\end{array}$ & $\begin{array}{l}\text { Equation in the } \\
\text { Phase Space }\end{array}$ & $\begin{array}{l}\text { Equation of the } \\
\text { Trajectories }\end{array}$ & $\begin{array}{c}\text { Simulation } \\
\text { Method }\end{array}$ \\
\hline $\begin{array}{l}\text { Discrete } \\
\text { Solvent }\end{array}$ & $\begin{array}{c}\left\{\mathbf{r}_{\text {solvent }}, \mathbf{p}_{\text {solvent }}\right. \\
\left.\mathbf{r}_{\text {solute }}, \mathbf{p}_{\text {solute }}\right\}\end{array}$ & Liouville & Newton & $\begin{array}{l}\text { Molecular } \\
\text { Dynamics }\end{array}$ \\
\hline \multirow{2}{*}{$\begin{array}{c}\text { Continuous } \\
\text { Solvent } \\
m_{\text {solvent }}<<m_{\text {solute }}\end{array}$} & $\left\{\mathbf{r}_{\text {solute }}, \mathbf{p}_{\text {solute }}\right\}$ & Fokker-Planck & Langevin & $\begin{array}{c}\text { Langevin } \\
\text { Brownian Dynamics }\end{array}$ \\
\hline & $\begin{array}{c}\left\{\mathbf{r}_{\text {solute }}\right\} \\
t>\tau_{\mathrm{V}}\end{array}$ & Smoluchowski & $\begin{array}{c}\text { Ermak } \\
\text { Algorithm }\end{array}$ & $\begin{array}{c}\text { Smoluchowski } \\
\text { Brownian Dynamics }\end{array}$ \\
\hline
\end{tabular}

Fig. 3 - Various levels of description for the description of the dynamics of ions in solution.

the ions. Indeed in that case, the ionic velocities have the time to relax and it can be shown (Van Kampen 1985) that they are actually fast variables. The dynamics of the ions is then given by the Smoluchowski equation, which only depends on the position of the particles.

A few works have been made at the Langevin level of description (Turq et al. 1977), but almost all theories correspond to the Smoluchowski level of approximation (Altenberger and Friedman 1983, Sung and Friedman 1983, Dufrêche et al. 2005a). The reason is that the characteristic time of ions in bulk solution is the Debye time of the electrolyte (Turq et al. 1992). This time is typically of a few nanoseconds, it is much longer than the relaxation time of the velocities (closer to the picosecond) so that the Smoluchowski approach is valid.

In the case of monohydrated clays the situation is completely different because of the presence of the mineral layers. The characteristic time of the motion of the ion is not the Debye time, because the ion diffusion is actually driven by the surface-ions interactions (averaged over the solvent configuration). Molecular dynamics simulations (Malikova et al. 2004) show that the dynamics follows a hopping mechanism (site-site diffusion), with a characteristic time close to the picosecond, which is of the order of magnitude of $\tau_{\mathrm{v}}$. Thus, contrary to bulk solutions, the Smoluchowski's equation is not valid. The correct Brownian model is based on the Langevin level of description.

There is another price to pay compared to the bulk case. The site-site diffusion is coupled to the hydration/dehydration of the water. Thus, it is not possible anymore to consider the shell of water molecules as a part of a Brownian particle, because the solvation changes during the diffusion process. As a matter of fact, Brownian theory can only be applied only to real heavy ions. The case of small ones cannot be studied from Brownian models, because the time scale of the hydration process is close to the time scale of diffusion.

\section{LANGEVIN Dynamics Simulation}

We applied a Langevin description to the case of a clay with $\mathrm{Cs}^{+}$counterions and a water monolayer. The separation of timescales between the slow solute (ion) and fast solvent (water) dynamics is such that one can 
average over the solvent degrees of freedom. Within the Langevin approach, the solute/solvent interaction is modelled by two parameters. The dynamical properties depend on a single friction coefficient $\gamma\left(\right.$ in $^{-1}$ ): The friction force on an ion is proportional to its velocity $\left(\mathbf{F}_{\text {solv }}=-m \gamma \mathbf{v}\right)$. The important point is that $\gamma$ is different from the bulk values because the solvent configuration is completely modified by the geometry in the monolayer. In particular, the orientational configuration of the water molecules is modified by the sheets so that the dielectric part of the friction (Hubbard and Onsager 1977, Bagchi 1998), which is an important contribution to the bulk friction, is completely different. The second parameter is the external (free energy) potential $V_{\text {eff }}$ which models the solute/surface interactions.

The Langevin equation which gives the evolution of the velocity $\mathbf{v}$ is

$$
\frac{\mathrm{d} \mathbf{v}}{\mathrm{d} t}=-\gamma \mathbf{v}+\frac{\mathbf{F}}{m}+\Gamma(t)
$$

where $\mathbf{F}$ is the external force, i.e. the opposite of the gradient of $V_{\text {eff }} . \Gamma(t)$ is a random force. It can be modelled by a white noise whose correlation function is related to $\gamma$ (Risken 1996). We solved this equation numerically using a first order algorithm. Such a numerical solution method is called the Langevin dynamics simulation method.

The Langevin equation is equivalent to the FokkerPlanck (FP) equation

$$
\partial_{t} f+\mathbf{v} \cdot \nabla f=\nabla_{\mathbf{v}} \cdot\left(\gamma \mathbf{v}+\frac{\nabla V}{m}+\frac{\gamma k_{B} T}{m} \nabla_{\mathbf{v}}\right) f
$$

which gives the evolution of the probability density function $f(\mathbf{x}, \mathbf{v}, t)$ of finding an ion at a given position with a given velocity. We also solved numerically the FP equation by a Lattice Fokker-Planck method (Moroni et al. 2006) which we used in this particular context of ionic mobility in compacted clays (Rotenberg et al. 2006). The two methods (Langevin dynamics and Lattice Fokker-Planck simulations) were found to give exactly the same results.

An important issue is the value of the McMillan potential $V_{\text {eff }}$ (McMillan and Mayer 1945), averaged over the configurations of the solvent. It is exactly defined as the $N$ particles distribution function of the solute when it is infinitely dilute. For the sake of sim- plicity, we performed preliminary calculations where $V_{\text {eff }}$ is given by the low density limit:

$$
V_{e f f}(\mathbf{r})=-k_{\mathrm{B}} T \ln \rho(\mathbf{r})
$$

with $\rho(\mathbf{r})$ is the average concentration of counterions obtained by molecular dynamics. This simplified model neglects the correlation among the ions.

$V_{e f f}$, which was assumed to be a 2D potential is represented in Figure 4. This potential clearly indicates that we have a site-site diffusion for the dynamics of the cesium ions, the depth of the potential being a few times the thermal energy $k_{\mathrm{B}} T$. Langevin dynamics gives exactly the same ion distribution (not represented). It is a direct consequence of the fact that the canonical distribution function is a stationary solution of the FokkerPlanck equation.

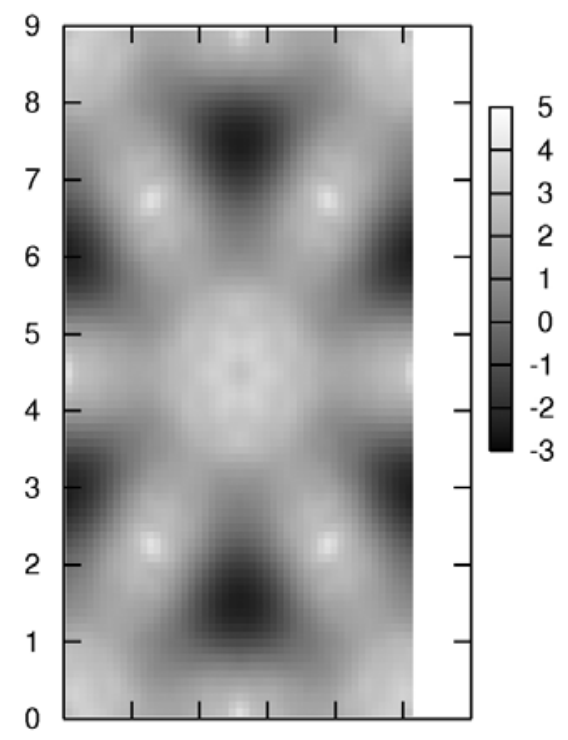

Fig. 4 - Effective potential ( $k_{\mathrm{B}} T$ units) for the counterions in clays obtained from molecular dynamics simulations. The dark and the white part respectively correspond to the position of the $\mathrm{Si}$ and $\mathrm{O}$ atoms of the surface of the clay sheets. The length unit is $\AA$.

\section{DIFFUSION OF IONS}

We calculated the velocity correlation function $Z(t)$ (Hansen and McDonald 1986) which characterizes the diffusion of the ions in the system for different values of the friction $\gamma$. The results are presented in Figure 5.

At large friction $\gamma=10 \mathrm{ps}^{-1}, Z(t)$ can be divided into two parts. First at short times a simple relaxation corresponds to the friction of the solvent. Then there 
is a long time negative tail which corresponds to the jumps from site to site. If the friction decreases and approaches $\gamma=1 \mathrm{ps}^{-1}$, oscillations appear in the velocity correlation function. They correspond to the oscillations of the ions in the layer sites. They are typical of the Langevin regime for which the velocity relaxation is not faster than the characteristic time of the interactions. The two times are close to $1 \mathrm{ps}$.

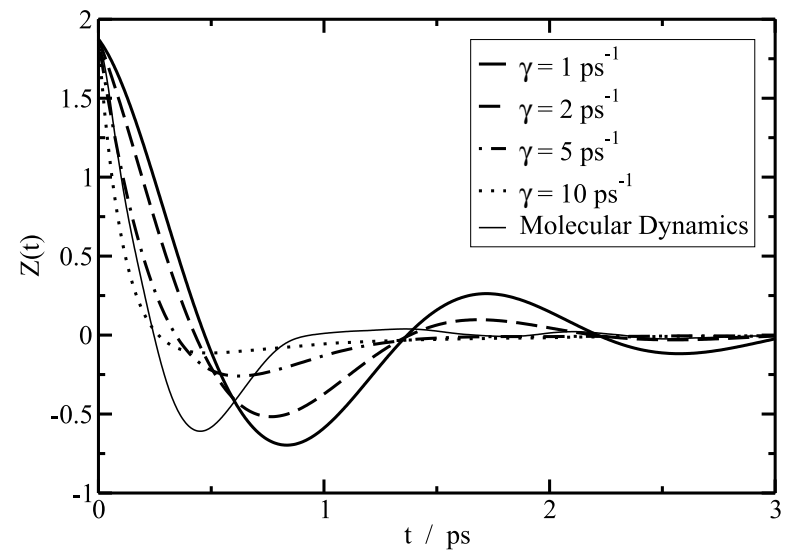

Fig. 5 - Velocity correlation function of the ions obtained from molecular dynamics (atomic simulations with explicit water molecules) and from Langevin dynamics dimulations for 4 values of the friction (continuous solvent simulations).

The results obtained from Langevin dynamics can be compared to the $Z(t)$ calculated from molecular dynamics. Of course, the velocity correlation function is quantitatively different because of the simplicity of this preliminary model: the friction is isotropic and the ion correlations are neglected. However the curves are qualitatively similar. Discrete solvent simulations recover small oscillations. The value of the friction can be obtained at small time scales. $\gamma$ seems to be between 2 and $5 \mathrm{ps}^{-1}$. It corresponds to a domain where $Z(t)$ starts to exhibit very small velocity oscillations.

\section{CONCLUSION}

We briefly describe the different approaches used to model the dynamics of clays. Every level of description is valid if the variables it models (relevant variables) are slow variables. In the case of hydrodynamics, the macroscopic laws are valid as long as the typical size of the system is larger than a few nanometers, if proper boundary conditions are taken into account. For monohydrated systems where there is just one layer of solvent molecules it is still possible to use a continuous solvent model if the ion is heavy. Then the dynamics is obtained from a Brownian model. Preliminary results indicate that this method may be applied for $\mathrm{Cs}^{+}$.

\section{RESUMO}

A teoria de transporte em meios porosos tais como argilas depende do nível de descrição. Na escala macroscópica, equações da hidrodinâmica são utilizadas. Tais descrições a nível do contínuo são convenientes para tratar o movimento do fluido em sistemas confinados. No entanto, tais equações são válidas se os poros do material são muito maiores do que as moléculas das componentes do sistema. Uma outra abordagem consiste em usar descrições moleculares. Esses dois métodos que correspondem a diferentes níveis de descrição são complementares. A ligação entre eles pode ser elucidada usando um procedimento de mudança de escala onde são tomadas médias das leis microscópicas sobre as variáveis rápidas para se obter as leis macroscópicas para tempos longos. Apresentamos esta abordagem no caso de argilas. Primeiramente apresentamos em detalhes os vários níveis de descrição bem como as relações entre eles, enfatizando o domínio de validade das equações hidrodinâmicas. Em seguida, focamos no caso de argilas desidratadas onde a hidrodinâmica não é relevante. Mostramos que é possível derivar um modelo simples para o movimento dos íons césio baseado na diferença entre as escalas de tempo do solvente e das partículas do soluto.

Palavras-chave: argila, modelagem multiescala, dinâmica molecular, hidrodinâmica, movimento Browniano.

\section{REFERENCES}

Ailawadi NK, Rahman A And Zwanzig R. 1971. Generalized hydrodynamics and analysis of current correlation functions. Phys Rev A 4: 1616-1625.

Altenberger AR AND Friedman HL. 1983. Theory of conductance and related isothermal transport coefficients in electrolytes. J Chem Phys 78: 4162-4173.

Arab M, Bougeard D AND SMIRnov KS. 2004. Structure and dynamics of interlayer species in a hydrated Zn-vermiculite. A molecular dynamics study. Phys Chem Chem Phys 6: 2446.

BAGCHI B. 1998. Microscopic derivation of the HubbardOnsager-Zwanzig expression of imiting ionic conductivity. J Chem Phys 109: 3989-3993.

BENCO L ET AL. 2001. Ab initio density functional theory applied to the structure and proton dynamics of clays. Chem Phys Lett 333: 479. 
BOEK ES AND SPRIK M. 2003. Ab initio molecular dynamics study of the hydration of a sodium smectite clay. J Phys Chem B 107: 3251.

DE Groot SR AND MAZur P. 1983. Non-Equilibrium Thermodynamics. Dover.

DUFrÊCHE J-F, BERNARD O, DURAND-VIDAL S AND TURQ P. 2005a. Analytical theories of transport in concentrated electrolyte solutions from the MSA. J Phys Chem B 109: 9873.

DUfrêche J-F, MARry V, MaLikova N AND TURQ P. 2005b. Molecular hydrodynamics for electro-osmosis in clays: from Kubo to Smoluchowski. J Mol Liq 118: 145.

Greathouse JA, Refson K and Sposito GM. 2000. Molecular dynamics simulation of water mobility in magnesium-smectite hydrates. J Am Chem Soc 122: 11459.

HANSEn J-P ANd MCDonald IR. 1986. Theory of Simple Liquids. Academic Press.

HubBard J AND OnSAger L. 1997. Dielectric dispersion and dielectric friction in electrolyte solutions. I. J Chem Phys 67: 4850.

Joly L, YBert C, TRIZAC E AND BocQuet L. 2006. Liquid friction on charged surfaces: From hydrodynamic slippage to electrokinetics. J Chem Phys 125: 204716.

Karaborni S, Smit B, Heidung W, Urai J And van OORT E. 1996. The swelling of clays: molecular simulations of the hydration of montmorillonite. Science 271: 1102.

LEROY P AND REVIL A. 2004. A triple layer model of the surface electrochemical properties of clay minerals. J Coll Int Science 270: 371.

Malikova N, Marry V, Dufrêche J-F And Turq P. 2004. Temperature effect in a montmorillonite clay at low hydration-microscopic simulation. Mol Phys 102: 1965.

MARRY V AND TURQ P. 2003. Microscopic simulations of interlayer structure and dynamics in bihydrated heteroionic montmorillonites. J Phys Chem B 107: 1832.

MARry V, Dufrêche J-F, JARdAT M AND TuRQ P. 2003. Equilibrium and electrokinetic phenomena in charged porous media from microscopic and mesoscopic models: electro-osmosis in montmorillonite. Mol Phys 101: 3111.

MCMillan WG And MaYer JE. 1945. The statistical mechanicsof multicomponents systems solutions. J Chem Phys 13: 276.

Moroni D, Rotenberg B, Hansen J-P, Succi S And MelchionnA S. 2006. Solving the Fokker-Planck kinetic equation on a lattice. Phys Rev E 73: 066707.

MOYNE C AND MURAD MA. 2002. Electro-chemo-mechanical couplings in swelling clays derived from a micro/ macro-homogenization procedure. Int $\mathrm{J}$ Solids Struct 39: 6159.

Moyne C And Murad MA. 2003. Macroscopic behavior of swelling porous media derived from micromechanical analysis. Transport in Porous Media 50: 127.

ONSAGER L AND FUOSS RM. 1932. Irreversible processes in electrolytes. J Phys Chem 36: 2689-2778.

Porion P, Al Mukhtar M, Faugere AM, Pellend RJ, Meyer S AND Delville A. 2003. Water self-diffusion within nematic dispersions of nanocomposites: a multiscale analysis of $1 \mathrm{H}$ pulsed gradient spin-echo $\mathrm{nmr}$ measurements. J Phys Chem B 107: 4012-4023.

RÉSIBOIS PMV. 1968. Electrolyte Theory. Harper \& Row.

RISKEN H. 1996. The Fokker-Planck Equation: Methods of Solutions and Applications. Springer Series in Synergetics.

Rosanne M, Paszkuta M, Thovert JF And Adler P. 2004. Electroosmotic coupling in compact clays. Geophys Research Lett 31: L18614.

Rotenberg B, Dufrêche J-F, Bagchi B, Giffaut E, HANSEN J-P AND TURQ P. 2006. Ion dynamics in compacted clays: Derivation of a two-state diffusion-reaction scheme from the lattice Fokker-Planck equation. J Chem Phys 14: 154701.

Skipper NT, Sposito G AND Chang F-RC. 1995. Clays Clay Miner 43: 294.

SUnG W AND Friedman HL. 1983. Time-correlation functions of the Smoluchowski level of description of solutions and suspensions. J Chem Phys 80: 2735-2741.

TRIZAC E ET AL. 2000. Effective interactions and phase behaviour for a model clay suspension in an electrolyte. J Phys Cond Matt 122: 11459.

Tunega D, Gerzabek MH And Lischka H. 2004. $A b$ initio molecular dynamics study of a monomolecular water layer on octahedral and tetrahedral kaolinite surfaces. J Phys Chem B 108: 5930.

TURQ P, LANTELME F AND FRIEDMAN HL. 1977. Brownian dynamics: its application to ionic solutions. J Chem Phys 66: 3039-3044.

Turq P, BARThel J AND Chemla M. 1992. Transport, Relaxation and Kinetic Processes in Electrolyte Solutions. Springer-Verlag.

VAN KAMPEN NG. 1985. Elimination of fast variables. Phys Rep 124: 69.

VAn Kampen NG. 1992. Stochastic Processes in Physics and Chemistry. North-Holland.

ZWANZIG R. 2001. Nonequilibrium Statistical Mechanics. Oxford University Press. 